\title{
EXTENT OF SERVICES SUPPORTED BY Q-SIGNALING OVER IP
}

QSIG is a signaling protocol used for interconnection of various telecommunication systems in corporate networks. These networks are composed of homogeneous or heterogeneous elements. Basic procedures of connection and supplementary services are transferred through messages, which are defined in recommendations ETSI or ISO. QSIG can be used for transport proprietary signaling between the same PBXS and is supported in voice gateway of solution of Voice over IP too and environment of IP network is often chosen for interconnection between PBXS. The main aim of this paper is to acquaint specialists with the result of the QSIG compatibility test between Siemens and Cisco. Siemens produces one of the most used telecommunication systems in the Czech republic and Cisco systems routers are the most used in IP solutions. In order to provide all supplementary services in telecommunication, Siemens developed a new protocol, called CorNet-NQ, which is a superset of QSIG and uses messages tunnelling through QSIG. For realization of this test the QSIG version PSSIV2 in agreement with the ISO standards was used. [1]

\section{Introduction}

QSIG is a signaling protocol for controlling the establishment, maintenance and clearing of calls between PINXs, nodal entity known as Private Integrated services Network eXchange. It provides an extremely powerful method of connecting PINX equipment in a corporate network. QSIG is not a proprietary standard. It is an open, international standard and is supported by the world's leading PBX suppliers. Twelve of the world's leading PBX manufacturers signed a Memorandum of Understanding (MoU) concerning the development and support of QSIG. The MoU, which came into effect on 1st February 1994, commits the signatories to facilitate the performance of interoperability tests and to:

- incorporate a Primary Rate interface (as defined in ETS 300 011) into its products,

- support a Basic Call (as defined in ETS 300 172),

- implement the generic procedures (as defined in ETS 300 239),
- implement QSIG supplementary services as far as each signatory considers to be economically viable,

- and participate in interoperability testing with the other signatories.

QSIG standards are developed within ECMA in Technical Committee 32 (TC32) for Communications, networks and systems interconnection. TC32 began to work on QSIG in 1988 and most of these standards were published also as ISO/IEC International Standards, endorsed by ETSI as European Standards and implemented by all major PBX vendors. [2]

\section{QSIG protocol stack}

QSIG standards specify a signaling system at the "Q" reference point, which is primarily intended for use on a common channel;

\begin{tabular}{|c|c|c|c|c|}
\hline Layer & \multicolumn{3}{|l|}{ Standards } & Description \\
\hline Layer 4-7 & \multicolumn{3}{|c|}{ Application mechanism } & $\begin{array}{l}\text { End to End protocol } \\
\text { network transparent }\end{array}$ \\
\hline \multirow[t]{3}{*}{ Layer 3} & \multicolumn{3}{|c|}{ Standards depend on the value of supplementary services } & $\begin{array}{l}\text { QSIG procedures for } \\
\text { supplementary services }\end{array}$ \\
\hline & \multicolumn{3}{|c|}{ ISO/IEC 11582, ETS300 239, ECMA165 } & $\begin{array}{l}\text { QSIG Generic Functional } \\
\text { Procedures }\end{array}$ \\
\hline & \multicolumn{3}{|c|}{ ISO/IEC 11574/11572, ETS300 171/172, ECMA142/143 } & QSIG Basic Call \\
\hline Layer 2 & \multicolumn{3}{|c|}{ ECMA141, ETS300 402} & \multirow{3}{*}{$\begin{array}{l}\text { Interface-dependent } \\
\text { protocols }\end{array}$} \\
\hline Layer 1 & $\begin{array}{l}\text { Basic Access (BRA) } \\
\text { ETS300 } 011 \\
\text { I.430 }\end{array}$ & $\begin{array}{l}\text { Primary Access (PRA) } \\
\text { ETS300 } 012 \\
\text { I. } 431\end{array}$ & & \\
\hline Medium & Copper Wire & Copper Wire & Optical fibre & \\
\hline
\end{tabular}

Figure 1: Reference model QSIG

\footnotetext{
* Miroslav Voznak

Technical University of Ostrava, Faculty of Electrical Engineering and Computer Science, Department of Electronics and Telecommunications, Tel: +420 596991699, E-mail: miroslav.voznak@vsb.cz
} 
e.g. G.703 interface. Within the public ISDN the two end PINXs are connected through two reference points using different ISDN protocols, namely DSS1 at the "T" reference point and ISUP within the public ISDN at the "N" reference point. For private ISDNs, only one protocol is necessary as the QSIG protocols have sufficient functionality to be used both within the network at transit nodes and on the outside at access nodes.

The architecture of QSIG signaling protocol agrees with the architecture RM OSI, see Figure 1.

The standard for Basic Call is ECMA 143 (ISO/IEC 11572), QSIG generic support for supplementary services is defined in ECMA-165 as a toolkit on which signaling for support of supplementary services can easily be built (ISO/IEC 11582). The QSIG protocol stack is identical in structure to the DSS1 protocol stack. Both follow the ISO reference model. Both can have an identical layer 1 and layer 2 (LAPD). However, at layer 3 QSIG and DSS 1 differ.

In the last years significant part of work inside TC32 is concerned with the interoperation of Private Integrated Services Networks (PISN) with IP Networks, in particular with the two following aspects:

- Interworking of PISNs and IP networks via Gateway, primarily between QSIG and H.323 and between QSIG and SIP,

- Connection of PISN components via IP network infrastructures (i.e. QSIG tunnelling).

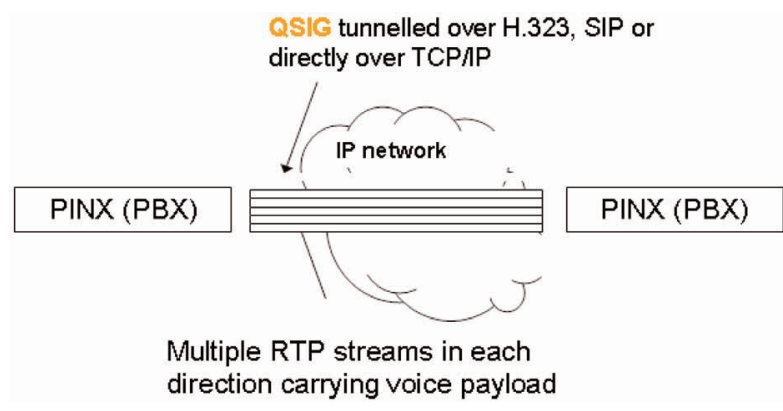

Figure 2: Tunnelling QSIG over IP network.

Inside the TC32 a new Task Group TG-17 was founded, known as TC32 - TG17, for standardization in the area of IP-based multimedia communications. This group considers possible future work in the area of NGN (Next Generation Networks), where close cooperation with ETSI is anticipated.

New ECMA standards allow tunnelling QSIG over IP network, see Figure 2, these standards are listed below.

Interworking between QSIG and H.323:

- ECMA-332 - basic call

- ECMA-307 - generic support for supplementary services

- ECMA-308 - call transfer supplementary services
- ECMA-309 - call diversion supplementary services

- ECMA-326 - call completion supplementary services

Interworking between QSIG and SIP:

- ECMA-339 - basic call

- work in progress on call transfer and call diversion services

Tunnelling of QSIG over IP (for PBX interconnection):

- ECMA-333 - tunnelling of QSIG over H.323

- ECMA-336 - tunnelling of QSIG directly over TCP/IP

- work in progress on tunnelling QSIG over SIP

Figure 3 shows that for private ISDNs, the QSIG protocols have sufficient functionality to be used both within the network at transit nodes and on the outside at access nodes. Hence QSIG is used between all three PINXs. The "Q" reference point is the logical signaling point between two PINXs. The physical connection to the PINXs is made at the " $\mathrm{C}$ " reference point. The Intervening Network (IVN) can be either dedicated channels (analogue or digital) or switched connections (for Virtual Private Networks).

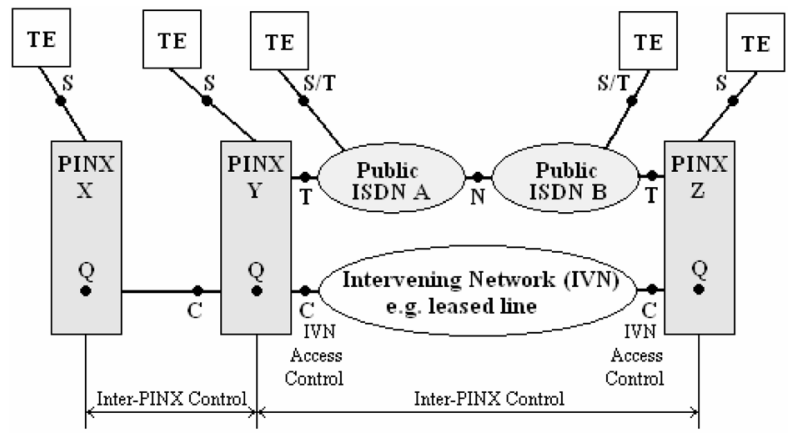

Figure 3: Reference model extended for corporate networks

\section{Conditions of QSIG test interoperability}

User signaling services can be divided into two groups:

- BASIC CALL - to transfer the control information required for the set-up, monitoring and release of connection and the identification of a subscriber's number,

- SUPPLEMENTARY SERVICES - to exchange signaling information for the control of the supplementary services and additional network features (ANFs)

System components used in test

Tab. 1

\begin{tabular}{|l|l|}
\hline PBX model & Siemens HiPath4000 \\
\hline PBX Release & Version 1-09 \\
\hline Signaling & QSIG (PSS1V2/ISO) \\
\hline Interface & ISDN BRI \\
\hline Voice gateway & Cisco 1751-V \\
\hline Gateway release & IOSTM 12.3(6a) \\
\hline VoX protocol & ITU-T H.323 \\
\hline
\end{tabular}


In this QSIG test interoperability individual supplementary services were tested and signalling messages were traced between interconnected equipment in all signalling path. [3], [4], [5], [6].

\section{Test configuration}

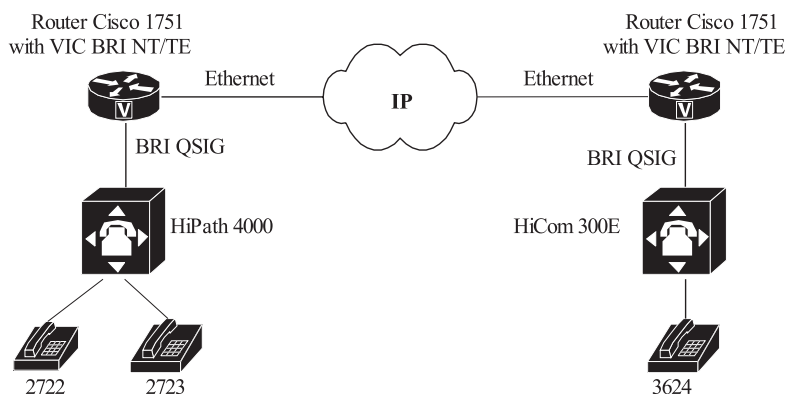

Figure 4: Used model of tested network.

\subsection{Configuration of the voice gateway}

Set up Notes

The Cisco 1751-V gateway with BRI-ISDN supports protocol QSIG, when the value of Switch-type parameter is set to basic-qsig. The network side on the BRI is set by the following commands:

- isdn layer1-emulate network,

- isdn protocol-emulate network.

Significant part of configuration in Cisco voice gateway is shown below as an example.

below as an example.
${ }^{*}$ for interface BRI

${ }^{*}$ for outgoing and incoming route

interface BRI0/0

no ip address

isdn switch-type basic-qsig

isdn overlap-receiving T302 5000

isdn protocol-emulate network

isdn layer1-emulate network

isdn incoming-voice voice

isdn static-tei 0

isdn timeout-signaling

voice-port 0/0,

compand-type a-law

cptone $\mathrm{CZ}$

bearer-cap $3100 \mathrm{~Hz}$

!

\subsection{Configuration of the PBX}

Set up Notes

The Siemens HiPath 4000 supports the protocol QSIG when the value of parameter Protvar in TDCSU command is set to PSSIV2, and the user side on the BRI is set by the following parameters:

- MASTER = N,

- $S M D=N$.

A significant part of configuration in Siemens HiPath 4000 is shown below as an example.

\author{
dial-peer voice 2 pots \\ destination-pattern ... \\ direct-inward-dial \\ port $0 / 0$ \\ dial-peer voice 11 voip \\ destination-pattern 36. \\ session target \\ ipv4:195.113.113.156 \\ codec g711alaw
}

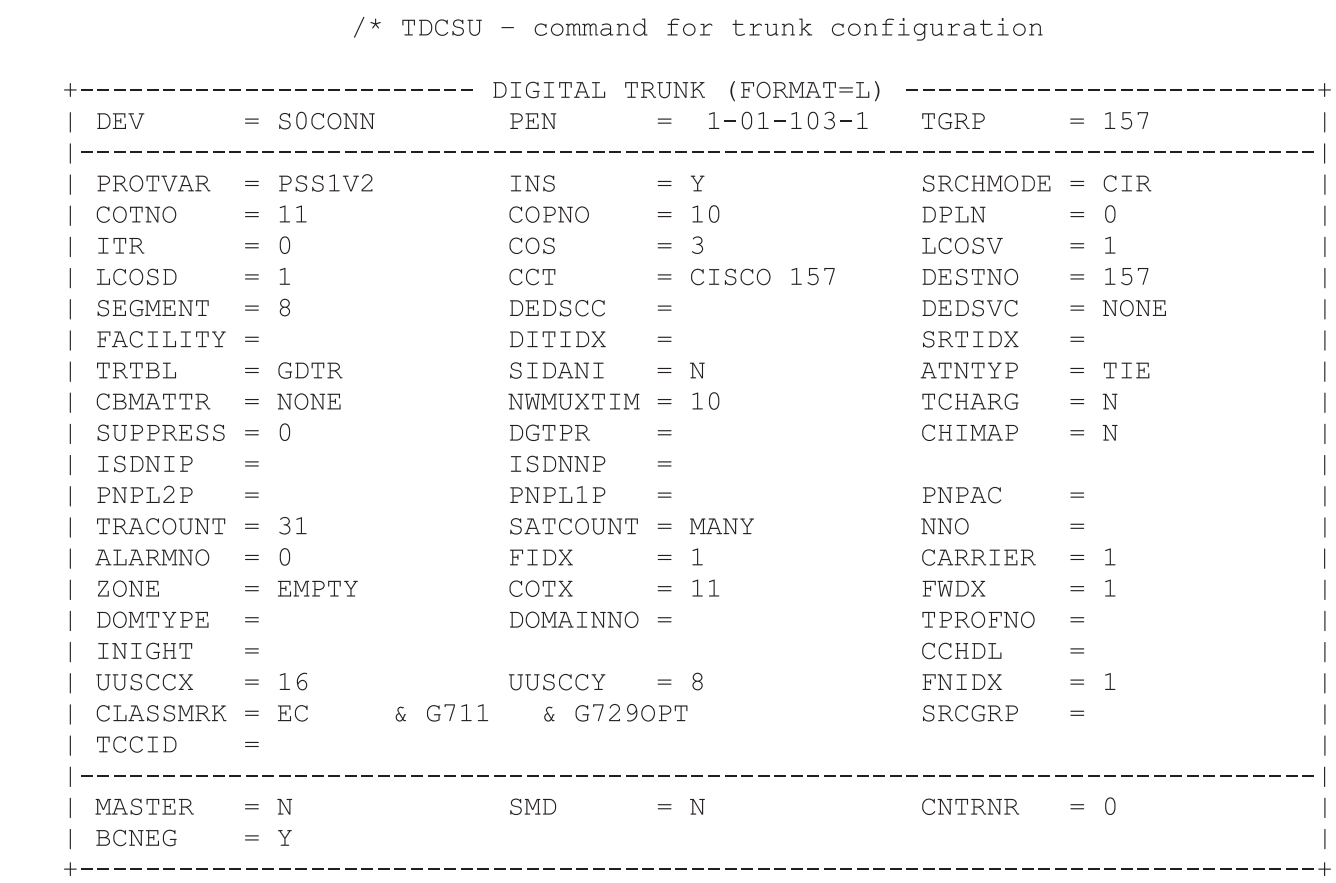

/* next commands RICHT, LDAT and WABE are needed, closed numbering scheme was used in configuration 


\subsection{Decoding of QSIG}

To decode the QSIG messages, SW application the WinVisu was used. It supports CORNET-NQ decoding protocol too and all the contents of signaling are in detail displayed in accordance with the recommendation of the chosen protocol, see Figure 5.

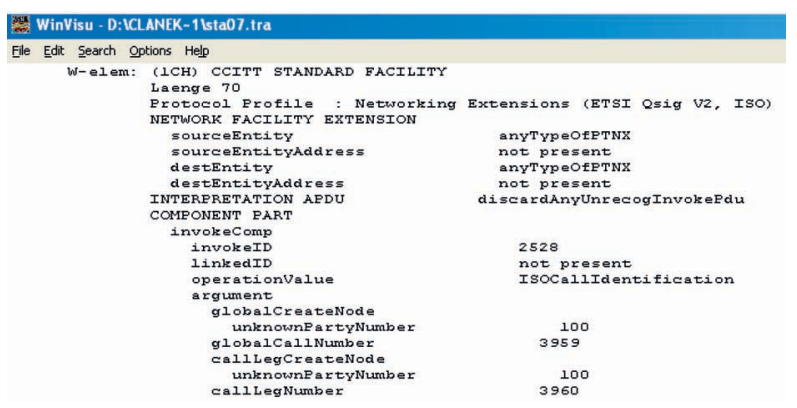

Fig. 5. Screen of WinVisu window

\section{Test result}

In the table below, the tested supplementary services are listed including the results related to each of these services.

Only one service (of all 22 supplementary services), Call Completion Busy Subscriber - CCBS did not work properly because the SETUP message was not transferred correctly through the IP network.

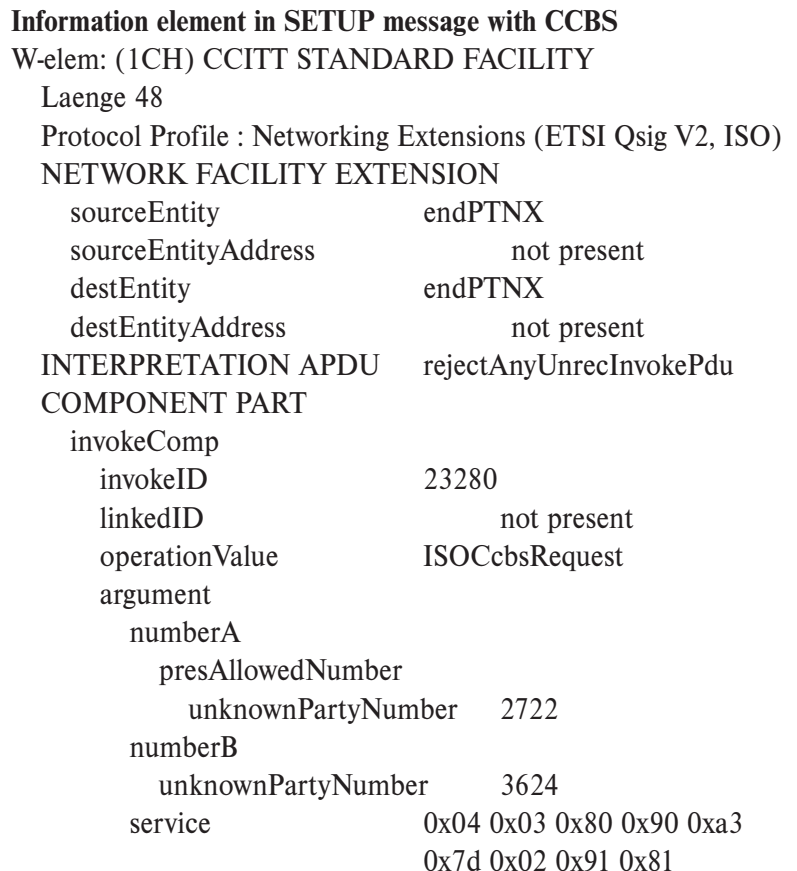

Table of tested supplementary services

Table 1

\begin{tabular}{|l|l|l|}
\hline Description & Busy subscriber - CCBS & NOK \\
\hline \multirow{2}{*}{ Call Completion } & No Reply - CCNR & OK \\
\hline \multirow{3}{*}{ Call Forwarding } & Busy subscriber - CFBS & OK \\
\cline { 2 - 3 } & No Reply - CFNR & OK \\
\cline { 2 - 3 } & Unconditional - CFU & OK \\
\hline Call Intrusion - CI & CLIP & OK \\
\hline Identification & COLP & OK \\
\cline { 3 - 3 } & CNIP & OK \\
\cline { 3 - 3 } & CONP & OK \\
\hline Identification Restriction & CLIR & OK \\
\cline { 3 - 3 } & COLR & OK \\
\hline Conference - CONF & OK \\
\hline Call Transfer - CT & OK \\
\hline Call Waiting - CW & OK \\
\hline Do Not Disturb - DND & OK \\
\hline Do Not Disturb Override - DNDO & OK \\
\hline HOLD & OK \\
\hline Message Waiting Indication - MWI & OK \\
\hline Direct Dialing In- DDI & OK \\
\hline Multiple subscriber number - MSN & OK \\
\hline Advice of charge - AOC D/E & OK \\
\hline Call Deflection - CD & OK \\
\hline Path Replacement - PR & Not tested \\
\hline Subadressing - SUB & Not tested \\
\hline User-User Signaling - UUS & Not tested \\
\hline
\end{tabular}

This supplementary service was not transferred because the voice gateway incorrectly processed the CCBS signaling request in SETUP message. On the other hand, the CCNR - Call Completion to Subscriber (in idle state) was transferred properly as it was indicated through MWI facility (Message Waiting Indication). Supplementary CD service was not tested as it is not supported by HiPath 4000 . 


\section{References:}

[1] ISO/IEC Standards, http://www.iso.ch/iso/en/ittf/PubliclyAvailableStandards

[2] ETS 300 172, Inter-exchange signaling protocol, ETS Institute, France, 1996

[3] PETERS J., DAVIDSON J.: Voice over IP Fundamentals, Cisco Press, Indianopolis USA, 2000, ISBN 1-57870-168-6

[4] VOZNAK, M.: Comparison of H.323 and SIP Protocol Specification, International Conference "Research in Telecommunication Technology 2003”, STU Bratislava, September 2003, p. 45-47, ISBN 80-227-1934-X

[5] HiPath 4000 Service Manual, Siemens AG, Munich, 2002, P31003-H3101-K104-2-20

[6] Cisco Systems Inc., http://www.cisco.com 